\title{
ENDPOINT ESTIMATES FOR COMMUTATORS OF CALDERÓN-ZYGMUND TYPE OPERATORS
}

\author{
ZongGuang LiU AND ShanZHEN LU*
}

\begin{abstract}
In this paper, we establish some endpoint estimates for the commutator, $[b, T]$, of a class of Calderón-Zygmund type operator, such as the weak type $L \log L$ estimate, weak type $\left(H^{1}, L^{1}\right)$ estimate and some estimates in the Hardy type spaces associated with $b$, where $b \in \operatorname{BMO}\left(\boldsymbol{R}^{n}\right)$.
\end{abstract}

\section{Introduction}

Calderón-Zygmund operators and their generalizations on Euclidean space $\boldsymbol{R}^{n}$ have been extensively studied [1-4]. In particular, Yabuta [3] introduced certain $\theta$ type Calderón-Zygmund operators to facilitate his study of certain classes of pseudo-differential operator. In this paper, we study the commutator of the following so-called $\theta$ type Calderón-Zygmund operator.

Definition 1. Let $\theta$ be a non-negative non-decreasing function on $\boldsymbol{R}^{+}$with $\int_{0}^{1} \theta(t) t^{-1}|\log t| d t<\infty$. A measurable function $K$ on $\boldsymbol{R}^{n} \times \boldsymbol{R}^{n} \backslash\left\{(x, x): x \in \boldsymbol{R}^{n}\right\}$ is said to be a $\theta$ type kernel if it satisfies

(i) $|K(x, y)| \leq C|x-y|^{-n}$ for $x \neq y$;

(ii) $|K(x, y)-K(z, y)|+|K(y, x)-K(y, z)| \leq \frac{C \theta(|x-z| /|x-y|)}{|x-y|^{n}}$,
for $|x-z|<|x-y| / 2$.

Let $T$ be a linear operator from $\mathscr{S}\left(\boldsymbol{R}^{n}\right)$ into its dual $\mathscr{S}^{\prime}\left(\boldsymbol{R}^{n}\right)$. We say $T$ is a $\theta$ type Calderón-Zygmund operator if

(1) $T$ can be extended to be a bounded linear operator on $L^{2}\left(\boldsymbol{R}^{n}\right)$;

(2) There is a $\theta$ type kernel $K$ such that $T f(x)=\int_{\text {supp } f} K(x, y) f(y) d y$ for all $f \in C_{0}^{\infty}\left(\boldsymbol{R}^{n}\right)$ and for all $x \notin \operatorname{supp} f$, where $C_{0}^{\infty}\left(\boldsymbol{R}^{n}\right)$ is the space of all infinitely differentiable functions on $\boldsymbol{R}^{n}$ with compact supports.

\footnotetext{
* Shanzhen Lu was partially supported by the National 973 Project Foundation of China. MR (1991) Subject Classification: 42B20, 35J05.

Key words: Calderón-Zygmund type operator, commutator, Hardy type space, $\operatorname{BMO}\left(\boldsymbol{R}^{n}\right)$.

Received June 11, 2001; revised November 26, 2001.
} 
Remark 1. The $\theta$ type Calderón-Zygmund operator introduced in Definition 1 is a special case of operator which is introduced by Yabuta, so the results for Yabuta's operator also hold for our operator. The following lemma is a result in [4].

Lemma 1. Let $\theta$ be a non-negative non-decreasing function on $\boldsymbol{R}^{+}$with $\int_{0}^{1} \theta(t) t^{-1} d t<\infty$. Let $T$ be a $\theta$ type Calderón-Zygmund operator. Then the following conditions are equivalent:

(1) $\int_{Q}|T a(x)| d x \leq C\|a\|_{L^{\infty}\left(\boldsymbol{R}^{n}\right)}$ for $a \in L^{\infty}\left(\boldsymbol{R}^{n}\right)$ with supp $a \subset Q$, a cube in $\boldsymbol{R}^{n}$;

(2) $T$ is a bounded operator from $H^{1}\left(\boldsymbol{R}^{n}\right)$ to $L^{1}\left(\boldsymbol{R}^{n}\right)$;

(3) $T$ is a bounded operator from $L_{0}^{\infty}\left(\boldsymbol{R}^{n}\right)$ to $\operatorname{BMO}\left(\boldsymbol{R}^{n}\right)$;

(4) $T$ is a bounded operator from $L^{q}\left(\boldsymbol{R}^{n}\right)$ to $W L^{q}\left(\boldsymbol{R}^{n}\right)$ for some $q \in(1, \infty)$;

(5) $T$ is a bounded operator on $L^{q}\left(\boldsymbol{R}^{n}\right)$ for some $q \in(1, \infty)$;

(6) $T$ is weak type $(1,1)$.

In this paper, we establish some endpoint estimates for commutator of the $\theta$ type Calderón-Zygmund operator:

$$
[b, T] f(x)=b(x) T f(x)-T(b f)(x),
$$

where $b \in \operatorname{BMO}\left(\boldsymbol{R}^{n}\right)$.

Most the notation that we use is standard. $Q$ will always denote a cube with sides parallel to the axes, $\lambda Q(\lambda>0)$ denotes the cube $Q$ dilated by $\lambda$. For a locally integrable function $f, f_{Q}$ denotes the average of $f$ on $Q: f_{Q}=$ $(1 /|Q|) \int_{Q} f(y) d y$.

As usual, a function $A:[0, \infty) \rightarrow[0, \infty)$ is said to be a Young function if it is continuous, convex and increasing and satisfying $A(0)=0, A(t) \rightarrow \infty$ as $t \rightarrow \infty$. We define the $A$-average of a function $f$ over a cube $Q$ by means of the following Luxemberg norm:

$$
\|f\|_{A, Q}=\inf \left\{\lambda>0: \frac{1}{|Q|} \int_{Q} A\left(\frac{|f(y)|}{\lambda}\right) d y \leq 1\right\} .
$$

The generalized Hölder inequality

$$
\frac{1}{|Q|} \int_{Q}|f(y) g(y)| d y \leq\|f\|_{A, Q}\|g\|_{\bar{A}, Q}
$$

holds, where $\bar{A}$ be the complementary Young function associated to $A$.

It is well known that $\bar{A}(t) \approx \exp t$ with $A(t)=t\left(1+\log ^{+} t\right)$. The maximal function associated to $A(t)=t\left(1+\log ^{+} t\right)$ was defined as

$$
M_{L \log L} f(x)=\sup _{x \in Q}\|f\|_{A, Q} .
$$

The maximal function associated to $A(t)=t$ is the well-known Hardy-Littlewood maximal function. For $\delta>0$, we define the $\delta$-maximal function as $M_{\delta}(f)=$ $\left[M\left(|f|^{\delta}\right)\right]^{1 / \delta}$ and the $\delta$-Sharp maximal function as

$$
M_{\delta}^{\sharp}(f)=\left[M^{\sharp}\left(|f|^{\delta}\right)\right]^{1 / \delta},
$$

where $M^{\sharp}$ be the well-known Fefferman-Stein’s Sharp maximal function: 


$$
M^{\sharp} f(x)=\sup _{x \in Q} \frac{1}{|Q|} \int_{Q}\left|f(y)-f_{Q}\right| d y .
$$

Following the results in [6], the $L^{p}(1<p<\infty)$ boundedness for commutator $[b, T]$ is the corollary of the following Sharp function estimates. Then

Lemma 2. Let $T$ be a $\theta$ type Calderón-Zygmund operator and $1<p<\infty$.

$$
M^{\sharp}(T f)(x) \leq C M_{p} f(x) .
$$

Proof. For any $x \in \boldsymbol{R}^{n}$ and any cube $Q$ with $x \in Q$, let $x_{0}$ be the centre of $Q$, and

$$
f=f \chi_{2 Q}+f \chi_{\boldsymbol{R}^{n} \backslash 2 Q}=f_{1}+f_{2} .
$$

By the $L^{p}$ boundedness of $T$ and the Hölder inequality, we have

$$
\frac{1}{|Q|} \int_{Q}\left|T f_{1}(y)\right| d y \leq\left(\frac{1}{|Q|} \int_{Q}\left|T f_{1}(y)\right|^{p} d y\right)^{1 / p} \leq C\left(\frac{1}{|Q|} \int_{2 Q}|f(y)|^{p} d y\right)^{1 / p} \leq C M_{p} f(x) \text {. }
$$

When $y \in Q$, we apply the condition of $\theta$ to get that

$$
\begin{aligned}
\left|T f_{2}(y)-T f_{2}\left(x_{0}\right)\right| & \leq \int_{\boldsymbol{R}^{n} \backslash 2 Q}\left|K(y, z)-K\left(x_{0}, z\right)\right||f(z)| d z \\
& \leq C \sum_{j=1}^{\infty} \int_{2^{j+1} Q \backslash 2^{j} Q} \frac{\theta\left(\left|y-x_{0}\right| /\left|z-x_{0}\right|\right)}{\left|z-x_{0}\right|^{n}}|f(z)| d z \\
& \leq C \sum_{j=1}^{\infty} \theta\left(2^{-j}\right) \frac{1}{\left|2^{j+1} Q\right|} \int_{2^{j+1} Q}|f(z)| d z \\
& \leq C \sum_{j=1}^{\infty} \theta\left(2^{-j}\right)\left(\frac{1}{\left|2^{j+1} Q\right|} \int_{2^{j+1} Q}|f(z)|^{p} d z\right)^{1 / p} \\
& \leq C \int_{0}^{1} \theta(t) t^{-1} d t M_{p} f(x) \leq C M_{p} f(x) .
\end{aligned}
$$

This implies that

$$
\frac{1}{|Q|} \int_{Q}\left|T f_{2}(y)-T f_{2}\left(x_{0}\right)\right| d y \leq C M_{p} f(x) .
$$

Thus, we obtain that

$$
M^{\sharp}(T f)(x) \leq C M_{p} f(x) .
$$

This completes the proof of Lemma 2 .

\section{Weak type $L \log L$ estimates and weak type $\left(H^{1}, L^{1}\right)$ estimates}

In this section, we establish firstly the weak type $L \log L$ estimates for $[b, T]$ by the method of the Sharp function estimates. Then we get the weak type $\left(H^{1}, L^{1}\right)$ estimates. Our main results are the following theorems 
THEOREM 1. Let $b \in \mathrm{BMO}\left(\boldsymbol{R}^{n}\right)$ and $T$ be a $\theta$ type Calderón-Zygmund operator. Then, there exists a positive constant $C$ such that for each smooth function $f$ with compact support and for all $\lambda>0$,

$$
\left|\left\{x \in \boldsymbol{R}^{n}:|[b, T] f(x)|>\lambda\right\}\right| \leq C\|b\|_{*} \int_{\boldsymbol{R}^{n}} \frac{|f(y)|}{\lambda}\left(1+\log ^{+} \frac{|f(y)|}{\lambda}\right) d y .
$$

Following the ideas of Pérez [9], we only need prove the following two Sharp function estimates.

Lemma 3. Let $b \in \operatorname{BMO}\left(\boldsymbol{R}^{n}\right), T$ be a $\theta$ type Calderón-Zygmund operator and $0<\delta<\varepsilon<1$. Then, there exists a positive constant $C=C_{\delta, \varepsilon}>0$ such that for each smooth function $f$ with compact support,

$$
M_{\delta}^{\sharp}([b, T] f)(x) \leq C\|b\|_{*}\left(M_{\varepsilon}(T f)(x)+M_{L \log L} f(x)\right) .
$$

Proof. Let $Q=Q(x, r)$ be an arbitrary cube. Since $0<\delta<\varepsilon<1$ implies $\left.|| \alpha\right|^{\delta}-|\beta|^{\delta}|\leq| \alpha-\left.\beta\right|^{\delta}$ for $\alpha, \beta \in \boldsymbol{R}$, it is enough to show for some complex constant $c=c_{Q}$ that there exists $C=C_{\delta}>0$ such that

$$
\left(\frac{1}{|Q|} \int_{Q}|[b, T] f(y)-c|^{\delta} d y\right)^{1 / \delta} \leq C\|b\|_{*}\left(M_{\varepsilon}(T f)(x)+M_{L \log L} f(x)\right) .
$$

Let $f=f \chi_{2 Q}+f \chi_{\boldsymbol{R}^{n} \backslash 2 Q}=f_{1}+f_{2}$. We write

$$
[b, T] f=\left(b-b_{2 Q}\right) T f-T\left(\left(b-b_{2 Q}\right) f_{1}\right)-T\left(\left(b-b_{2 Q}\right) f_{2}\right) .
$$

If we pick $c=c_{Q}=\left(T\left(\left(b-b_{2 Q}\right) f_{2}\right)\right)_{Q}$, we have

$$
\begin{aligned}
\left(\frac{1}{|Q|} \int_{Q}|[b, T] f(y)-c|^{\delta} d y\right)^{1 / \delta} & \\
\leq & C\left(\frac{1}{|Q|} \int_{Q}\left|b(y)-b_{2 Q}\right|^{\delta}|T f(y)|^{\delta} d y\right)^{1 / \delta}+C\left(\frac{1}{|Q|} \int_{Q}\left|T\left(\left(b-b_{2 Q}\right) f_{1}\right)(y)\right|^{\delta} d y\right)^{1 / \delta} \\
& +C\left(\frac{1}{|Q|} \int_{Q}\left|T\left(\left(b-b_{2 Q}\right) f_{2}\right)(y)-\left(T\left(\left(b-b_{2 Q}\right) f_{2}\right)\right)_{Q}\right|^{\delta} d y\right)^{1 / \delta} \\
= & I_{1}+I_{2}+I_{3} .
\end{aligned}
$$

To estimate $I_{1}$, we use the Hölder inequality with exponents $r$ and $r^{\prime}$ where $1<r<\varepsilon / \delta$ :

$$
\begin{aligned}
I_{1} & \leq C\left(\frac{1}{|Q|} \int_{Q}\left|b(y)-b_{2 Q}\right|^{\delta r^{\prime}} d y\right)^{1 / \delta r^{\prime}}\left(\frac{1}{|Q|} \int_{Q}|T f(y)|^{\delta r} d y\right)^{1 / \delta r} \\
& \leq C\|b\|_{*} M_{\delta r}(T f)(x) \leq C\|b\|_{*} M_{\varepsilon}(T f)(x) .
\end{aligned}
$$


Since $T$ is of weak type $(1,1)$ and $0<\delta<1$, the Kolmogorov inequality implies

$$
\begin{aligned}
I_{2} & \leq C|Q|^{-1} \frac{\left\|T\left(\left(b-b_{2 Q}\right) f_{1}\right) \chi_{Q}\right\|_{L^{\delta}\left(\boldsymbol{R}^{n}\right)}}{|Q|^{1 / \delta-1}} \leq C|Q|^{-1}\left\|T\left(\left(b-b_{2 Q}\right) f_{1}\right) \chi_{Q}\right\|_{W L^{1}\left(\boldsymbol{R}^{n}\right)} \\
& \leq C|Q|^{-1}\left\|\left(b-b_{2 Q}\right) f_{1}\right\|_{L^{1}\left(\boldsymbol{R}^{n}\right)} \leq C\left\|b-b_{2 Q}\right\|_{\exp L, 2 Q}\|f\|_{L \log L, 2 Q} \\
& \leq C\|b\|_{*} M_{L \log L} f(x) .
\end{aligned}
$$

In the last inequality, we use the estimate that $\left\|b-b_{Q}\right\|_{\exp L, Q} \leq C\|b\|_{*}$, it is equivalent to the inequality

$$
\frac{1}{|Q|} \int_{Q} \exp \left(\frac{\left|b(y)-b_{Q}\right|}{C\|b\|_{*}}\right) d y \leq C_{0}
$$

it is just a corollary of the well-known John-Nirenberg inequality. Then the Jensen inequality and the Fubini theorem yield

$$
\begin{aligned}
I_{3} \leq & \frac{C}{|Q|} \int_{Q}\left|T\left(\left(b-b_{2 Q}\right) f_{2}\right)(y)-\left(T\left(\left(b-b_{2 Q}\right) f_{2}\right)\right)_{Q}\right| d y \\
\leq & \frac{C}{|Q|^{2}} \int_{Q} \int_{Q} \int_{R^{n} \backslash 2 Q}|K(y, w)-K(z, w)|\left|\left(b(w)-b_{2 Q}\right) f(w)\right| d w d z d y \\
\leq & \frac{C}{|Q|^{2}} \int_{Q} \int_{Q} \sum_{j=1}^{\infty} \int_{2^{j+1} Q \backslash 2^{j} Q} \frac{\theta(|y-z| /|x-w|)}{|x-w|^{n}}\left|b(w)-b_{2 Q}\right||f(w)| d w d z d y \\
\leq & C \sum_{j=1}^{\infty} \theta\left(2^{-j}\right) \frac{1}{\left|2^{j+1} Q\right|} \int_{2^{j+1} Q}\left|b(w)-b_{2 Q}\right||f(w)| d w \\
\leq & C \sum_{j=1}^{\infty} \theta\left(2^{-j}\right) \frac{1}{\left|2^{j+1} Q\right|} \int_{2^{j+1} Q}\left|b(w)-b_{2^{j+1} Q}\right||f(w)| d w \\
& +C \sum_{j=1}^{\infty} \theta\left(2^{-j}\right)\left|b_{2^{j+1} Q}-b_{2^{j}} Q\right| \frac{1}{\left|2^{j+1} Q\right|} \int_{2^{j+1} Q}|f(w)| d w \\
\leq & C \sum_{j=1}^{\infty} \theta\left(2^{-j}\right)\left\|b-b_{2^{j+1}} Q\right\|_{\exp L, 2^{j+1} Q}\|f\|_{L \log L, 2^{j+1} Q}+C \sum_{j=1}^{\infty} j \theta\left(2^{-j}\right)\|b\|_{*} M f(x) \\
\leq & C \sum_{j=1}^{\infty} j \theta\left(2^{-j}\right)\|b\|_{*} M_{L \log L} f(x) \leq C \int_{0}^{1} \theta(t) t^{-1}|\log t| d t\|b\|_{*} M_{L \log L} f(x) \\
\leq & C\|b\|_{*} M_{L \log L} f(x) .
\end{aligned}
$$

This completes the proof of Lemma 3.

Using a similar method, we can establish the following Sharp function estimate and omit the details. 
Lemma 4. Let $0<\alpha<1$ and $T$ be a $\theta$ type Calderón-Zygmund operator. Then, for any $f \in C_{0}^{\infty}\left(\boldsymbol{R}^{n}\right)$ and $x \in \boldsymbol{R}^{n}$, there exists a constant $C=C_{\alpha}>0$, such that

$$
M_{\alpha}^{\sharp}(T f)(x) \leq C M f(x) .
$$

Now, we establish the weak type $\left(H^{1}, L^{1}\right)$ estimate for $[b, T]$.

Theorem 2. Let $b \in \mathrm{BMO}\left(\boldsymbol{R}^{n}\right)$ and $T$ be a $\theta$ type Calderón-Zygmund operator. Then the commutator $[b, T]$ is a bounded operator from $H^{1}\left(\boldsymbol{R}^{n}\right)$ to weak $L^{1}\left(\boldsymbol{R}^{n}\right)$, i.e. for any $\lambda>0$, there exists a constant $C>0$, such that

$$
\left|\left\{x \in \boldsymbol{R}^{n}:|[b, T] f(x)|>\lambda\right\}\right| \leq \frac{C}{\lambda}\|f\|_{H^{1}\left(\boldsymbol{R}^{n}\right)} .
$$

Proof. For any given $f \in H^{1}\left(\boldsymbol{R}^{n}\right)$, by atomic decomposition we get $f=$ $\sum_{j=1}^{\infty} \lambda_{j} a_{j}$, where each $a_{j}$ be a $(1, \infty, 0)$ atom with $\|f\|_{H^{1}\left(\boldsymbol{R}^{n}\right)}=\inf \left(\sum_{j=1}^{\infty}\left|\lambda_{j}\right|\right)$. We may assume that $f$ is a finite sum $\sum_{Q} \lambda_{Q} a_{Q}$ with $\sum_{Q}\left|\lambda_{Q}\right| \leq 2\|f\|_{H^{1}\left(\boldsymbol{R}^{n}\right)}$. Once Theorem 2 is proven for such $f$, for general $f$ is the limit of this kind of $f_{k}$ (in $H^{1}$ norm or almost everywhere sense) where $f_{k}$ are finite sums having forms of $\sum_{Q} \lambda_{Q} a_{Q}$, Theorem 2 follows by a limiting argument, using the $L^{2}$-boundedness of $[b, T]$. It is convenient for us to assume that each $Q$ (the supporting cube of $\left.a_{Q}\right)$ in the given atomic decomposition of $f$ is dyadic and $\lambda_{Q}>0$.

For fixed $\lambda>0$ and the finite collection of dyadic cube $Q$ and associated positive scalars $\lambda_{Q}>0$ in the given atomic decomposition of $f$, by Lemma 4.1 in [5], there exists a collection of pairwise disjoint dyadic cubes $S$ such that
(1) $\sum_{Q \subset S} \lambda_{Q} \leq 2^{n} \lambda|S|$, for all $S$;
(2) $\sum_{S}|S| \leq \lambda^{-1} \sum_{Q} \lambda_{Q}$;
(3)$$
\left\|\sum_{Q \not \subset \text { any } S} \lambda_{Q}|Q|^{-1} \chi_{Q}\right\|_{L^{\infty}\left(\boldsymbol{R}^{n}\right)} \leq \lambda .
$$

Denote $E=\bigcup_{S} 2 S$, then $|E| \leq C \lambda^{-1}\|f\|_{H^{1}\left(\boldsymbol{R}^{n}\right)}$.

Set $h(x)=\sum_{S} \sum_{Q \subset S} \lambda_{Q} a_{Q}$ and $g(x)=f(x)-h(x)$. By (3), $\|g\|_{L^{\infty}\left(\boldsymbol{R}^{n}\right)} \leq \lambda$ and the $L^{2}\left(\boldsymbol{R}^{n}\right)$ boundedness of $[b, T]$ implies

$$
\begin{aligned}
\left|\left\{x \in \boldsymbol{R}^{n} \backslash E:|[b, T] g(x)|>\lambda / 4\right\}\right| & \leq \frac{C}{\lambda^{2}}\|[b, T] g\|_{L^{2}\left(\boldsymbol{R}^{n}\right)}^{2} \leq \frac{C}{\lambda^{2}}\|g\|_{L^{2}\left(\boldsymbol{R}^{n}\right)}^{2} \\
& \leq \frac{C}{\lambda}\|g\|_{L^{1}\left(\boldsymbol{R}^{n}\right)} \leq \frac{C}{\lambda}\|f\|_{L^{1}\left(\boldsymbol{R}^{n}\right)} \leq \frac{C}{\lambda}\|f\|_{H^{1}\left(\boldsymbol{R}^{n}\right)} .
\end{aligned}
$$

Thus, we only need prove following inequality

$$
\left|\left\{x \in \boldsymbol{R}^{n} \backslash E:|[b, T] h(x)|>\lambda / 4\right\}\right| \leq \frac{C}{\lambda}\|f\|_{H^{1}\left(\boldsymbol{R}^{n}\right)} .
$$

For any fixed cube $Q=Q\left(x_{Q}, r_{Q}\right)$, by moments condition for $a_{Q}$ we have 


$$
\begin{aligned}
{[b, T] a_{Q}(x)=} & \int_{\boldsymbol{R}^{n}}(b(x)-b(y)) K(x, y) a_{Q}(y) d y \\
= & \int_{\boldsymbol{R}^{n}}\left(b(x)-b_{Q}\right)\left[K(x, y)-K\left(x, x_{Q}\right)\right] a_{Q}(y) d y \\
& +\int_{\boldsymbol{R}^{n}} K(x, y)\left(b_{Q}-b(y)\right) a_{Q}(y) d y .
\end{aligned}
$$

Since $x \in \boldsymbol{R}^{n} \backslash E$ implies that $x \in \boldsymbol{R}^{n} \backslash 2 Q$ for any cube $Q$ in the given atomic decomposition, by the smoothness condition of $\theta$ we get

$$
\begin{aligned}
|[b, T] h(x)| & \leq C \sum_{S} \sum_{Q \subset S} \lambda_{Q} \frac{\left|b(x)-b_{Q}\right| \theta\left(r_{Q} /\left|x-x_{Q}\right|\right)}{\left|x-x_{Q}\right|^{n}}+\left|\sum_{S} \sum_{Q \subset S} T\left(\left(b_{Q}-b\right) a_{Q}\right)(x)\right| \\
& =I_{1}(x)+I_{2}(x) .
\end{aligned}
$$

By the condition of $\theta(t)$, we obtain

$$
\begin{aligned}
\mid\{x \in & \left.\boldsymbol{R}^{n} \backslash E: I_{1}(x)>\lambda / 8\right\} \mid \\
& \leq \frac{C}{\lambda} \sum_{S} \sum_{Q \subset S} \lambda_{Q} \int_{\boldsymbol{R}^{n} \backslash 2 Q} \frac{\left|b(x)-b_{Q}\right| \theta\left(r_{Q} /\left|x-x_{Q}\right|\right)}{\left|x-x_{Q}\right|^{n}} d x \\
& \leq \frac{C}{\lambda} \sum_{S} \sum_{Q \subset S} \lambda_{Q} \sum_{l=1}^{\infty} \int_{2^{l+1} Q \backslash 2^{\prime} Q} \frac{\left|b(x)-b_{Q}\right| \theta\left(r_{Q} /\left|x-x_{Q}\right|\right)}{\left|x-x_{Q}\right|^{n}} d x \\
& \leq \frac{C}{\lambda} \sum_{S} \sum_{Q \subset S} \lambda_{Q} \sum_{l=1}^{\infty} \theta\left(2^{-l}\right) \frac{1}{\left|2^{l+1} Q\right|} \int_{2^{l+1} Q}\left|b(x)-b_{Q}\right| d x \\
& \leq \frac{C}{\lambda} \sum_{S} \sum_{Q \subset S} \lambda_{Q} \sum_{l=1}^{\infty} l \theta\left(2^{-l}\right)\|b\|_{*} \leq \frac{C\|b\|_{*}}{\lambda} \int_{0}^{1} \theta(t) t^{-1}|\log t| d t \sum_{S} \sum_{Q \subset S} \lambda_{Q} \\
& \leq \frac{C\|b\|_{*}}{\lambda}\|f\|_{H^{1}\left(\boldsymbol{R}^{n}\right)} .
\end{aligned}
$$

The weak type $(1,1)$ boundedness of $T$ implies the following estimate

$$
\begin{aligned}
\left|\left\{x \in \boldsymbol{R}^{n} \backslash E: I_{2}(x)>\lambda / 8\right\}\right| & \leq \frac{C}{\lambda} \sum_{S} \sum_{Q \subset S} \lambda_{Q}\left\|\left(b-b_{Q}\right) a_{Q}\right\|_{L^{1}\left(\boldsymbol{R}^{n}\right)} \\
& \leq \frac{C}{\lambda} \sum_{S} \sum_{Q \subset S} \lambda_{Q} \frac{1}{|Q|} \int_{Q}\left|b(y)-b_{Q}\right| d y \\
& \leq \frac{C\|b\|_{*}}{\lambda} \sum_{S} \sum_{Q \subset S} \lambda_{Q} \leq \frac{C\|b\|_{*}}{\lambda}\|f\|_{H^{1}\left(\boldsymbol{R}^{n}\right)} .
\end{aligned}
$$

This finishes the proof of Theorem 2 . 


\section{The estimates on Hardy type spaces}

It is well known that the commutator $[b, T]$ isn't a bounded operator from $H^{1}$ to $L^{1}$ even when $T$ is a usual Calderón-Zygmund operator, but it is a bounded operator from $H_{b}^{1}$ to $L^{1}$, where $H_{b}^{1}$ is a Hardy type space associated with $b \in \mathrm{BMO}\left(\boldsymbol{R}^{n}\right)$. In this section, we discuss this problem when $T$ is a $\theta$ type Calderón-Zygmund operator. Let us give some notations.

Definition 2. Let $b$ be a locally integrable function, $0<p \leq 1$. It is said that a bounded function $a$ is a $H_{b}^{p}\left(\boldsymbol{R}^{n}\right)$ atom if it satisfies

(1) supp $a \subset Q=Q\left(x_{0}, r\right)$ for some $r>0$;

(2) $\|a\|_{L^{\infty}\left(\boldsymbol{R}^{n}\right)} \leq|Q|^{-1 / p} ;$

(3) $\int_{\boldsymbol{R}^{n}} x^{\beta} a(x) d x=\int_{\boldsymbol{R}^{n}} x^{\beta} a(x) b(x) d x=0$ for any $|\beta| \leq[1 / p-1]$.

It is said that a temperate distribution $f$ belongs to $H_{b}^{p}\left(\boldsymbol{R}^{n}\right)$ if, in the $\mathscr{S}^{\prime}\left(\boldsymbol{R}^{n}\right)$ sense, it can be written as $f=\sum_{j=1}^{\infty} \lambda_{j} a_{j}$, where $a_{j}$ is a $H_{b}^{p}\left(\boldsymbol{R}^{n}\right)$ atom and $\sum_{j=1}^{\infty}\left|\lambda_{j}\right|^{p}<\infty$. We define on $H_{b}^{p}\left(\boldsymbol{R}^{n}\right)$ the quasinorm

$$
\|f\|_{H_{b}^{p}\left(\boldsymbol{R}^{n}\right)}=\inf \left(\sum_{j=1}^{\infty}\left|\lambda_{j}\right|^{p}\right)^{1 / p} .
$$

Theorem 3. Let $b \in \operatorname{BMO}\left(\boldsymbol{R}^{n}\right)$ and $T$ be a $\theta$ type Calderón-Zygmund operator, $0<p \leq 1$ and $\int_{0}^{1} \frac{\theta^{p}(t)|\log t|^{p}}{t^{(1-p) n+1}} d t<\infty$. Then the commutator $[b, T]$ is a bounded operator from $H_{b}^{p}\left(\boldsymbol{R}^{n}\right)$ to $L^{p}\left(\boldsymbol{R}^{n}\right)$.

Proof. By the condition of $\theta(t)$, it is easy to see that $\theta(t)<\theta^{p}(t)$ where $t \in(0, \varepsilon)$ for some $\varepsilon \in(0,1)$. This implies that

$$
\int_{0}^{1} \theta(t) t^{-1}|\log t| d t<C \int_{0}^{1} \frac{\theta(t)}{t^{(1-p) n+1}} d t \leq C \int_{0}^{1} \frac{\theta^{p}(t)|\log t|^{p}}{t^{(1-p) n+1}} d t .
$$

Thus we can use the results in Section 1, and get the $L^{q}(1<q<\infty)$ boundedness of $[b, T]$. Hence, as in the proof of Theorem 2, we only need to prove that, for any $H_{b}^{p}\left(\boldsymbol{R}^{n}\right)$ atom $a$, there exists a constant $C>0$ independent of $a$, such that $\int_{\boldsymbol{R}^{n}}|[b, T] a(x)|^{p} d x \leq C$.

Let $\operatorname{supp} a \subset Q=Q\left(x_{0}, r\right)$ and write

$$
\begin{aligned}
\int_{\boldsymbol{R}^{n}}|[b, T] a(x)|^{p} d x & \leq \int_{2 Q}|[b, T] a(x)|^{p} d x+\int_{\boldsymbol{R}^{n} \backslash 2 Q}|[b, T] a(x)|^{p} d x \\
& =J_{1}+J_{2} .
\end{aligned}
$$

Then, by the $L^{q}$ boundedness of $[b, T]$ and by the Hölder inequality, we have 


$$
\begin{aligned}
J_{1} & \leq|2 Q|^{1-p / q}\left(\int_{\boldsymbol{R}^{n}}|[b, T] a(x)|^{q} d x\right)^{p / q} \\
& \leq C|Q|^{1-p / q}\|a\|_{L^{q}\left(\boldsymbol{R}^{n}\right)}^{p} \leq C|Q|^{1-p / q}|Q|^{-1}|Q|^{p / q}=C .
\end{aligned}
$$

and

$$
J_{2} \leq \sum_{j=1}^{\infty} \int_{2^{j+1} Q \backslash 2^{j} Q}|[b, T] a(x)|^{p} d x \leq \sum_{j=1}^{\infty}\left|2^{j+1} Q\right|^{1-p}\left(\int_{2^{j+1} Q \backslash 2^{j} Q}|[b, T] a(x)| d x\right)^{p} .
$$

We write

$$
\begin{aligned}
\int_{2^{j+1} Q \backslash 2^{j} Q}|[b, T] a(x)| d x \leq & \int_{2^{j+1} Q \backslash 2^{j} Q}\left|b(x)-b_{Q}\right||T a(x)| d x \\
& +\int_{2^{j+1} Q \backslash 2^{j} Q}\left|T\left(\left(b-b_{Q}\right) a\right)(x)\right| d x \\
= & J_{21}+J_{22} .
\end{aligned}
$$

Since $2\left|y-x_{0}\right|<\left|x-x_{0}\right|$ when $y \in Q$ and $x \in 2^{j+1} Q \backslash 2^{j} Q$ with $j=1,2, \ldots$, we get

$$
\begin{aligned}
J_{21} & \leq \int_{2^{j+1} Q \backslash 2^{j} Q}\left|b(x)-b_{Q}\right| \int_{Q}\left|K(x, y)-K\left(x, x_{0}\right)\right||a(y)| d y d x \\
& \leq \int_{2^{j+1} Q \backslash 2^{j} Q}\left|b(x)-b_{Q}\right| \int_{Q} \frac{\theta\left(\left|y-x_{0}\right| /\left|x-x_{0}\right|\right)}{\left|x-x_{0}\right|^{n}}|a(y)| d y d x \\
& \leq C \theta\left(2^{-j}\right)|Q|^{1-1 / p} \frac{1}{\left|2^{j+1} Q\right|} \int_{2^{j+1} Q}\left|b(x)-b_{Q}\right| d x \\
& \leq C j \theta\left(2^{-j}\right)|Q|^{1-1 / p}\|b\|_{*} .
\end{aligned}
$$

Using the moment vanishing condition of $a$, we have

$$
\begin{aligned}
J_{22} & \leq \int_{2^{j+1} Q \backslash 2^{j} Q} \int_{Q}\left|K(x, y)-K\left(x, x_{0}\right)\right|\left|b(y)-b_{Q}\right||a(y)| d y d x \\
& \leq C \int_{2^{j+1} Q \backslash 2^{j} Q} \int_{Q} \frac{\theta\left(\left|y-x_{0}\right| /\left|x-x_{0}\right|\right)}{\left|x-x_{0}\right|^{n}}\left|b(y)-b_{Q}\right||a(y)| d y d x \\
& \leq C \theta\left(2^{-j}\right) \int_{2^{j+1} Q \backslash 2^{j} Q} \frac{d x}{\left|x-x_{0}\right|^{n}} \int_{Q}\left|b(y)-b_{Q}\right||a(y)| d y \\
& \leq C \theta\left(2^{-j}\right)|Q|^{1-1 / p} \frac{1}{|Q|} \int_{Q}\left|b(x)-b_{Q}\right| d x \\
& \leq C \theta\left(2^{-j}\right)|Q|^{1-1 / p}\|b\|_{*} .
\end{aligned}
$$


Thus we obtain

$$
\begin{aligned}
J_{2} & \leq C \sum_{j=1}^{\infty}\left|2^{j+1} Q\right|^{1-p}\left(j \theta\left(2^{-j}\right)|Q|^{1-1 / p}\|b\|_{*}\right)^{p} \\
& \leq C\|b\|_{*}^{p} \sum_{j=1}^{\infty} j^{p} 2^{j(1-p) n} \theta^{p}\left(2^{-j}\right) \leq C\|b\|_{*}^{p} \int_{0}^{1} \frac{\theta^{p}(t)|\log t|^{p}}{t^{(1-p) n+1}} d t \leq C\|b\|_{*}^{p} .
\end{aligned}
$$

This finishes the proof of Theorem 3.

Remark 2. In the case that $T$ is a usual Calderón-Zygmund operator, $\theta(t)=t^{\varepsilon}$ for some $\varepsilon>0$, we can see that the conditions of Theorem 3 hold with $n /(n+\varepsilon)<p \leq 1$. Thus the commutator $[b, T]$ is a bounded operator from $H_{b}^{p}\left(\boldsymbol{R}^{n}\right)$ to $L^{p}\left(\boldsymbol{R}^{n}\right)$ whenever $n /(n+\varepsilon)<p \leq 1$. This is the main result in [10].

Remark 3. When $p=1$, the conditions of $\theta(t)$ in Theorem 3 coincide with those in Theorem 2.

Acknowledgement. The authors would like to express their gratitude to the referee for his very valuable comments and suggestions.

\section{REFERENCES}

[ 1 ] J.-L. Journé, Calderón-Zygmund Operators, Pseudo-Differential Operators and the Cauchy Integral of Calderón, Lecture Notes in Math. 994, Springer-Verlag, Berlin, 1983.

[2] E. M. Stein, Harmonic Analysis: Real-Variable Methods, Orthogonality, and Oscillatory Integrals, Princeton Math. Ser. 43, Princeton University Press, Princeton, 1993.

[ 3 ] K. Yabuta, Generalizations of Calderón-Zygmund operators, Studia Math., 82 (1985), $17-$ 31.

[4] T. QUeK AND D. YANG, Calderón-Zygmund-type operators on weighted weak Hardy spaces over $\boldsymbol{R}^{n}$, Acta Math. Sin. (Engl. Ser.), 16 (2000), 141-160.

[ 5 ] M. Christ, Weak type $(1,1)$ bounds for rough operators, Ann. of Math. (2), 128 (1988), 19 42.

[6] J. Alvarez, R. J. Bagby, D. S. Kurtz and C. Pérez, Weighted estimates for commutators of linear operators, Studia Math., 104 (1993), 195-209.

[ 7 ] F. John and L. Nirenberg, On functions of bounded mean oscillation, Comm. Pure Appl. Math., 14 (1961), 415-426.

[8] C. Fefferman and E. M. Stein, $H^{p}$ spaces of several variables, Acta Math., 129 (1972), 137-193.

[ 9] C. PÉREZ, Endpoint estimates for commutators of singular integral operators, J. Funct. Anal., 128 (1995), 163-185.

[10] J. Alvarez, Continuity properties for linear commutators of Calderón-Zygmund operators, Collect. Math., 49 (1998), 17-31.

Department of Mathematics

BeIJING NoRMal University

BeIJING, 100875

P. R. China 